\title{
Whole-exome sequencing association studies on impaired spermatogenesis in different ethnic groups in Russia
}

\author{
Semyon K. Kolmykov \\ Institute of Cytology and Genetics \\ SB RAS \\ Novosibirsk, Russia \\ kolmykovsk@gmail.com \\ Maxim A. Kleshev \\ Institute of Cytology and Genetics \\ SB RAS \\ Novosibirsk, Russia \\ max82c11@bionet.nsc.ru
}

\author{
Gennadiy V.Vasiliev \\ Institute of Cytology and Genetics \\ SB RAS \\ Novosibirsk, Russia \\ genn@bionet.nsc.ru \\ Aleksandr V. Osadchuk \\ Institute of Cytology and Genetics \\ SB RAS \\ Novosibirsk, Russia \\ osadchuk@bionet.nsc.ru
}

\author{
Mikhail P. Ponomarenko \\ Institute of Cytology and Genetics \\ SB RAS \\ Novosibirsk, Russia \\ pon@bionet.nsc.ru \\ Ludmila V. Osadchuk \\ Institute of Cytology and Genetics \\ SB RAS \\ Novosibirsk, Russia \\ losadch@bionet.nsc.ru
}

\begin{abstract}
In this study we identified single nucleotide polymorphisms in whole-exome sequencing data for different ethnic groups in Russia: Slavs, Yakuts and Buryats, and investigated its associations with impaired spermatogenesis.
\end{abstract}

Keywords - WES, SNP, association analysis, impaired spermatogenesis, reproductive potential, infertility

\section{Introduction}

The decline in male reproductive potential, marked in various regions of the world, raises the question of the causes of this global phenomenon. Currently, there is a significant gap in our knowledge of the male reproductive potential in the Russian Federation, trends in its regional and ethnic variation and genetic control. Meanwhile, these issues become especially relevant in connection with increased attention to the demographic situation in the country and increased prevention of reproductive disorders.

In approximately $40 \%$ of infertile men, the etiology of infertility and subfertility remains unclear [1], and modern molecular genetic approaches, in particular, whole-exome sequencing (WES), expanding the possibilities of genome research, can reveal new genes involved in controlling male fertility. In this study we identified single nucleotide polymorphisms (SNPs) in whole-exome sequencing data for different ethnic groups and investigated its association with spermatogenesis defects.

\section{Materials And Methods}

\section{Study sample}

The study sample included 62 participants from 3 ethnic groups Slavs, Yakuts, and Buryats (see Tab. 1).

TABLE I. SPERMATOGENIC AND ETHNIC CHARACTERISTICS OF THE INVESTIGATED MALE SAMPLE

\begin{tabular}{|c|c|c|}
\hline Ethnic group & Normospermia, num & Pathospermia, num \\
\hline Yakuts & 10 & 9 \\
\hline Buryats & 10 & 9 \\
\hline Slavs & 12 & 12 \\
\hline All & 32 & 30 \\
\hline
\end{tabular}

\section{WES data processing and quality control}

The raw WES data were aligned to the reference genome (GRCh38) using BWA-MEM [2]. Optical and PCR duplicates were removed using Picard [3]. Further steps for detecting SNVs and INDELs were carried out using HaplotypeCaller according to GATK Best Practices [4]. Consequently, sets of variations were obtained (562018, 581274, and 519824 variations in the Yakut, Slavic, and Buryat cohorts, respectively).

The obtained variation sets were converted to PLINK format. Further quality control analysis was carried out using PLINK software [5]. At the first stage, poorly presented $(<2 \%$ of the number of samples) polymorphisms were filtered out, as well as ones deviating from the Hardy-Weinberg equilibrium with a threshold of 1e-6. Subsequently polymorphisms with a Minor Allele Frequency $<0.5 \%$ were removed. The heterozygosity rate of the samples participating in the study was also analyzed. According to the results, none of the samples deviated \pm 3 SD from the heterozygosity rate mean. As a result of the quality control, sets of 104113, 100202, and 90061 variants were selected in the representatives of the Yakut, Slavic, and Buryat populations, respectively for further analysis.

The analysis of the population structure of the obtained data sets yielded the values of Genomic control inflation factor $\lambda$ equal to $1.03745,1.00119$ and 1.06702 , which indicates a low level of population stratification of the studied samples.

Moreover, we applied the aforementioned pipeline to identify the set of single nucleotide polymorphisms for the jointed data set (62 patients: 32 patients with normospermia and 30 patients with patospermia). As a result, 103983 SNPs were obtained for further analysis of association.

\section{Association analysis}

An analysis of the association of identified polymorphisms with spermatogenesis defects was performed using $\chi 2$-test of association and logistic regression using PLINK [5].

\section{Results And Discussion}

According to the results of the association analysis, none of the associations reached Bonferroni-corrected significance levels (0.05 / number of SNPs): 4.8e-7, 4.99e-7, 5.55e-7 and 4.81e-7 for Slavs, Yakuts, Buryats and jointed groups respectively. A partial intersection of the top-20 lists of polymorphisms associated with impaired spermatogenesis was observed and analysed. In particular, the presence of an association of polymorphism in KLHL10 gene in the jointed group has been shown. An association of other SNPs in this gene with spermatogenesis disorders was shown previously [6]. For further more significant analysis of associations, it is planned to increase the size of the studied sample. 


\section{ACKNOWLEDGMENT}

This study was supported by the Russian Science Foundation, grant No. 19-15-00075.

\section{REFERENCES}

[1] Krausz C, Cioppi F, Riera-Escamilla A. Testing for genetic contributions to infertility: potential clinical impact. Expert review of molecular diagnostics. 2018 Apr 3;18(4):331-46.

[2] Li H. Aligning sequence reads, clone sequences and assembly contigs with BWA-MEM. arXiv preprint arXiv:1303.3997. 2013 Mar 16.

[3] Broad Institute. Picard Toolkit: Java command line tools for manipulating high-throughput sequencing (HTS) data and formats such as SAM/BAM/CRAM and VCF. http://broadinstitute.github.io/picard.
[4] Poplin R, Ruano-Rubio V, DePristo MA, Fennell TJ, Carneiro MO, Van der Auwera GA, Kling DE, Gauthier LD, Levy-Moonshine A, Roazen D, Shakir K. Scaling accurate genetic variant discovery to tens of thousands of samples. BioRxiv. 2018 Jan 1:201178.

[5] Chang CC, Chow CC, Tellier LC, Vattikuti S, Purcell SM, Lee JJ. Second-generation PLINK: rising to the challenge of larger and richer datasets. Gigascience. 2015 Dec 1;4(1):s13742-015.

[6] Yatsenko AN, Roy A, Chen R, Ma L, Murthy LJ, Yan W, Lamb DJ, Matzuk MM. Non-invasive genetic diagnosis of male infertility using spermatozoal RNA: KLHL10 mutations in oligozoospermic patients impair homodimerization. Human molecular genetics. 2006 Dec 1;15(23):3411-9. 